\title{
EFECTO DE LA SUPLEMENTACIÓN CON FUENTES DE COBRE SOBRE EL COMPORTAMIENTO PRODUCTIVO, MORFOMETRÍA INTESTINAL Y NIVEL DE COBRE HEPÁTICO EN POLLOS DE CARNE
}

\section{Effect of Supplementation with Copper Sources on Broiler Performance, InTESTinal Morphology AND Liver CopPer Content}

Otto Zea M. ${ }^{1,2,3}$, Carlos Vílchez P. ${ }^{1}$

\section{Resumen}

\begin{abstract}
El estudio tuvo por objetivo evaluar el efecto de la suplementación de cobre sobre el comportamiento productivo, los niveles de cobre en el hígado y la morfometría intestinal en pollos de 0 a 40 días de edad. Se emplearon 200 pollos BB machos de la Línea Cob500, distribuidos en cinco tratamientos con cuatro repeticiones y 10 animales por repetición: T1, dieta basal con zinc bacitracina (control positivo); T2, dieta basal sin antibiótico (control negativo); T3, dieta basal con sulfato de cobre; T4, dieta basal con oxicloruro de cobre; T5, dieta basal con cobre clorado tribásico (CCTB). El cobre suplementado fue de $250 \mathrm{mg} / \mathrm{kg}$. No se encontró diferencia estadística en peso corporal, ganancia de peso diario, rendimiento de carcasa y rendimiento económico. El índice de conversión y el consumo de alimento fueron mejores en T3 en relación a T1 y T2 ( $<<0.05)$. El mayor nivel de cobre en hígado fue en T5 ( $\mathrm{p}<0.05)$. Asimismo, la suplementación de cobre influyó la altura y área de vellosidad en el yeyuno comparado a los grupos control. Se concluye que con la suplementación de $250 \mathrm{ppm} \mathrm{Cu}$, proveniente del sulfato de cobre, se mejora la conversión alimenticia y que la suplementación de cobre, independientemente de la fuente, afecta la morfología intestinal.
\end{abstract}

Palabras clave: cobre, pollos de carne, hígado, vellosidades intestinales

\section{Abstract}

This study aimed to evaluate the effect of different copper sources on broiler performance, intestinal morphology and liver copper contents. Two hundred one-day old Cobb 500 male chicks were randomly distributed into five groups, using 4 replicates

\footnotetext{
${ }^{1}$ Departamento Académico de Nutrición, Facultad de Zootecnia, Universidad Nacional Agraria La Molina, Lima

${ }^{2}$ E-mail: angelozmz@ hotmail.com

${ }^{3}$ Dirección actual: Estación Experimental del Centro de Investigación IVITA, Universidad Nacional Mayor de San Marcos, Maranganí, Cusco
} 
and 10 birds per replicate. The treatments were: $\mathrm{T} 1$, basal diet containing antibiotic (positive control); T2, basal diet without antibiotic (negative control); T3, as T2 +250 ppm Cu from copper sulphate; $\mathrm{T} 4$, as $\mathrm{T} 2+250 \mathrm{ppm} \mathrm{Cu}$ from copper oxychloride; $\mathrm{T} 5, \mathrm{~T} 2+$ $250 \mathrm{ppm} \mathrm{Cu}$ from tribasic copper chloride. No significant differences were found between treatments on final body weight, daily body weight gain, cost/benefit ratio and carcass yield. The lowest feed intake and the best feed efficiency were observed in T2 as compared to T1 and T2 (p<0.05). The highest copper content in liver occurred in T5 $(\mathrm{p}<0.05)$. Copper supplements affected villus height and area in jejunum as compared to the control groups. It is concluded that supplementation with $250 \mathrm{ppm} \mathrm{Cu}$ from copper sulphate improves feed efficiency and that copper supplementation regardless the source affected intestinal morphology.

Key words: copper, broilers, liver, intestinal villus

\section{INTRODUCCIÓN}

Los promotores de crecimiento incluidos en la premezcla de la dieta de los pollos de carne permiten asegurar una eficiente conversión alimenticia y adecuada ganancia de peso (Colin et al., 1994). Los antibióticos son, por lo general, los principales promotores de crecimiento que se han venido empleando en la avicultura comercial (Martínez, 1996; Romero, 1998); no obstante, hay una creciente tendencia a disminuir su uso debido a que su empleo indiscriminado puede generar, a mediano plazo, resistencia a las enfermedades en la población humana (van den Bogaard y Stobberingh, 2000; Desmonts et al., 2004). Debido a esto, diversos trabajos de investigación están abocados en la búsqueda de alternativas al uso de antibióticos en la industria de producción animal.

El cobre, por otro lado, es un micromineral esencial debido a que interviene en numerosas actividades fisiológicas del ave como son el metabolismo del hierro, crecimiento y desarrollo del colágeno y en la hematopoyesis (Mejía et al., 2006). El requerimiento de cobre en broilers, según el NRC (1994), es de 8 $\mathrm{mg} / \mathrm{kg}$; no obstante, se ha determinado que cuando se agrega en la premezcla niveles mayores de los recomendados (125 a $250 \mathrm{mg} /$ $\mathrm{kg}$ ) presenta actividad como promotor de crecimiento y antimicrobiano (Luo et al., 2005; Miñón et al., 2006). Estos resultados han generado interés en el uso del cobre como alternativa al empleo de antibióticos para promover el crecimiento de las aves y mantener la salud del tracto gastrointestinal.

Es importante considerar que las características del cobre, así como de cualquier otro mineral, tales como tamaño de grano, solubilidad y potencial oxidativo de sus fuentes, pueden tener influencia sobre su biodisponibilidad y, por tanto, en su actividad sobre el animal y sus parámetros productivos (Ledoux et al., 1991). Se dispone de estudios que han determinado la fuente ideal del cobre así como la cantidad que se debería incorporar en la dieta animal (Miles et al., 1998).

En el mercado existen diversos productos que se usan como fuente de cobre. Entre ellos destacan productos orgánicos e inorgánicos como el sulfato de cobre, el cobre clorado tribásico (CCTB) y el oxicloruro de cobre (Pesti y Bakalli, 1998; Brainer et al., 2003). El sulfato de cobre es el modelo estándar de utilización de cobre; sin embargo, presenta una gran variabilidad sobre su efecto en el crecimiento de las aves (Abaza et al., 2009). Por otro lado, existe poca información sobre el uso del CCTB y el oxicloruro de cobre como fuentes de cobre, y del efecto de comparar estas fuentes a nivel experimental en las condiciones ambientales del país para su uso comercial. Además, debido a que 
el cobre tiene influencia sobre el sistema gastrointestinal (Xia et al., 2004; Arias y Koutsos, 2006), se podría evaluar su actividad mediante el análisis morfométrico de las vellosidades intestinales, así como del contenido de cobre en el hígado de los animales.

Basado en estas informaciones, el propósito del presente trabajo fue evaluar la suplementación con diferentes fuentes de cobre sobre el comportamiento productivo, citomorfometría intestinal y nivel de cobre hepático en pollos de carne.

\section{Materiales y MéTodos}

El presente estudio se llevó a cabo en el Laboratorio de Investigación en Nutrición y Alimentación de Aves (LINAA) de la Universidad Nacional Agraria de La Molina (UNALM), Lima. Se emplearon 200 pollos BB de la Línea Cobb 500, machos de un día de edad. Las aves fueron distribuidas al azar en 5 tratamientos de 40 pollos cada uno. Cada tratamiento constó de 4 repeticiones con 10 pollos por repetición. El tiempo de crianza fue de 1 a 10 días de edad para la fase de inicio, de 11 a 22 días de edad para la fase de crecimiento y de 23 a 40 días de edad para la fase de acabado. Las aves estuvieron alojadas en dos jaulas metálicas de malla galvanizada (baterías) de cinco pisos y con dos divisiones por piso, y con comederos y bebederos lineales tipo canaleta en la parte externa.

Los tratamientos fueron: $\mathrm{T} 1$, dieta basal con antibiótico (control positivo); T2, dieta basal sin antibiótico (control negativo); T3, $\mathrm{T} 2+250 \mathrm{ppm} \mathrm{Cu}$ como sulfato de cobre; $\mathrm{T} 4$, $\mathrm{T} 2+250 \mathrm{ppm} \mathrm{Cu}$ como oxicloruro de cobre, y T5, T2 + 250 ppm Cu como cloruro de cobre tribásico (CCTB). Las dietas experimentales fueron formuladas siguiendo las especificaciones nutricionales de la Línea Cobb 500 (Cobb-Vantress, 2002). La composición $\mathrm{y}$ valor nutricional calculado de la dieta basal se muestran en el Cuadros 1,2 y 3.
El contenido de cobre en el alimento se determinó por espectrofotometría de absorción atómica en el Laboratorio de Certificaciones del Perú S.A. (CERPER). Se registró semanalmente el peso vivo, consumo de alimento y conversión alimenticia, tomando en consideración los animales que murieron durante el experimento. $\mathrm{Al}$ final del periodo de crianza (40 d) se sacrificaron ocho animales por tratamiento para determinar el rendimiento de carcasa y se tomaron muestras para la determinación del contenido de cobre en el hígado, y para evaluar la citomorfología intestinal. La determinación del contenido de cobre en el hígado se realizó mediante espectrofotometría de absorción atómica, utilizando la metodología de la Norma Oficial Mexicana (NOM-117-SSA1-1994).

Para las mediciones citomorfométricas se colectaron tres segmentos del intestino delgado de $2 \mathrm{~cm}$ cada uno. El primer segmento se ubicó al inicio del duodeno (duodeno craneal), el segundo a $2 \mathrm{~cm}$ de la salida del colédoco (duodeno caudal) y el tercero correspondió al yeyuno, el cual se ubicó a 8 $\mathrm{cm}$ antes del divertículo de Meckel. Las muestras fueron guardadas en formol al $10 \%$ y remitidas al Laboratorio de Patología de la Facultad de Medicina Veterinaria de la Universidad Nacional Mayor de San Marcos, Lima. Las muestras fueron procesadas siguiendo los métodos histológicos convencionales (fijación con formol al 10\%, deshidratación/aclaramiento, inclusión en parafina, cortes al micrótomo y tinción con hematoxilina y eosina) (Banks, 1996). Las mediciones se hicieron siguiendo una adaptación del protocolo de evaluación utilizado por Batista de Oliveira et al. (2000), que consistió en colocar tres cortes seriados en lugar de cuatro por segmento y en realizar 15 mediciones citomorfométricas como mínimo por lámina histológica, en lugar de 30.

La medida altura de vellosidad fue la distancia tomada a partir de la región basal, que coincide con la porción superior de las criptas hasta el ápice de la vellosidad. El grosor de las vellosidades fue medido en el pun- 
Cuadro 1. Composición porcentual y valor nutritivo de las dietas experimentales empleadas en la etapa de inicio (0 a 10 días)

\begin{tabular}{|c|c|c|c|c|c|}
\hline & \multicolumn{5}{|c|}{ Tratamientos ${ }^{1}$} \\
\hline & $\mathrm{T} 1$ & $\mathrm{~T} 2$ & $\mathrm{~T} 3$ & $\mathrm{~T} 4$ & T5 \\
\hline Maíz & 59.40 & 59.40 & 59.40 & 59.40 & 59.40 \\
\hline Torta de soya & 28.92 & 28.92 & 28.92 & 28.92 & 28.92 \\
\hline Harina de pescado & 4.00 & 4.00 & 4.00 & 4.00 & 4.00 \\
\hline Aceite de soya & 3.00 & 3.00 & 3.00 & 3.00 & 3.00 \\
\hline Fosfato dicálcico & 1.74 & 1.74 & 1.74 & 1.74 & 1.74 \\
\hline Subproducto de trigo & 1.23 & 1.23 & 1.23 & 1.23 & 1.23 \\
\hline Carbonato de calcio & 0.70 & 0.70 & 0.70 & 0.70 & 0.70 \\
\hline Sal común & 0.40 & 0.40 & 0.40 & 0.40 & 0.40 \\
\hline DL Metionina & 0.19 & 0.19 & 0.19 & 0.19 & 0.19 \\
\hline $\begin{array}{l}\text { Premezcla vitaminas + } \\
\text { minerales }\end{array}$ & 0.10 & 0.10 & 0.10 & 0.10 & 0.10 \\
\hline Cloruro de colina 60 & 0.10 & 0.10 & 0.10 & 0.10 & 0.10 \\
\hline $\begin{array}{l}\text { Adsorvente de } \\
\text { micotoxinas }\end{array}$ & 0.10 & 0.10 & 0.10 & 0.10 & 0.10 \\
\hline Coccidiostato & 0.05 & 0.05 & 0.05 & 0.05 & 0.05 \\
\hline $\mathrm{HCl}$ Lisina & 0.03 & 0.03 & 0.03 & 0.03 & 0.03 \\
\hline Antioxidante & 0.02 & 0.02 & 0.02 & 0.02 & 0.02 \\
\hline Antibiótico & 0.05 & - & - & - & - \\
\hline Sulfato de cobre & - & - & 0.10 & - & - \\
\hline Oxicloruro de cobre & - & - & - & 0.05 & - \\
\hline ССТВ & - & - & - & - & 0.05 \\
\hline \multicolumn{6}{|c|}{ Valor Nutricional (Calculado) } \\
\hline $\begin{array}{l}\text { Energía metabolizable, } \\
\mathrm{kcal} \mathrm{kg}^{-1}\end{array}$ & 2,988 & 2,988 & 2,988 & 2,988 & 2,988 \\
\hline Proteín a bruta, \% & 21.00 & 21.00 & 21.00 & 21.00 & 21.00 \\
\hline Grasa total, $\%$ & 6.16 & 6.16 & 6.16 & 6.16 & 6.16 \\
\hline Fibra cruda, $\%$ & 2.81 & 2.81 & 2.81 & 2.81 & 2.81 \\
\hline Calcio, $\%$ & 1.00 & 1.00 & 1.00 & 1.00 & 1.00 \\
\hline Fósforo disponible, $\%$ & 0.50 & 0.50 & 0.50 & 0.50 & 0.50 \\
\hline Lisina total, $\%$ & 1.20 & 1.20 & 1.20 & 1.20 & 1.20 \\
\hline Metionina total, $\%$ & 0.56 & 0.56 & 0.56 & 0.56 & 0.56 \\
\hline Met + Cis total, $\%$ & 0.89 & 0.89 & 0.89 & 0.89 & 0.89 \\
\hline Treonina total, $\%$ & 0.81 & 0.81 & 0.81 & 0.81 & 0.81 \\
\hline Triptófano, \% & 0.24 & 0.24 & 0.24 & 0.24 & 0.24 \\
\hline Sodio, \% & 0.20 & 0.20 & 0.20 & 0.20 & 0.20 \\
\hline
\end{tabular}

1 T1: dieta basal con antibiótico (control positivo), T2: dieta basal sin antibiótico (control negativo), T3: T2 + 250 ppm Cu como sulfato de cobre, T4: T2 + 250 ppm Cu como oxicloruro de cobre, T5: $\mathrm{T} 2+250$ ppm Cu como cloruro de cobre tribásico (CCTB) 
Cuadro 2. Composición porcentual y valor nutritivo de las dietas experimentales empleadas en la etapa de crecimiento (11 a 22 días)

\begin{tabular}{|c|c|c|c|c|c|}
\hline & \multicolumn{5}{|c|}{ Tratamientos $^{2}$} \\
\hline & $\mathrm{T} 1$ & $\mathrm{~T} 2$ & $\mathrm{~T} 3$ & $\mathrm{~T} 4$ & T5 \\
\hline Maíz & 62.57 & 62.57 & 62.57 & 62.57 & 62.57 \\
\hline Torta de soya & 23.52 & 23.52 & 23.52 & 23.52 & 23.52 \\
\hline Harina de pescado & 4.00 & 4.00 & 4.00 & 4.00 & 4.00 \\
\hline Aceite de soya & 4.00 & 4.00 & 4.00 & 4.00 & 4.00 \\
\hline Fosfato dicálcico & 1.66 & 1.66 & 1.66 & 1.66 & 1.66 \\
\hline Subproducto de trigo & 2.57 & 2.57 & 2.57 & 2.57 & 2.57 \\
\hline Carbonato de calcio & 0.68 & 0.68 & 0.68 & 0.68 & 0.68 \\
\hline Sal común & 0.32 & 0.32 & 0.32 & 0.32 & 0.32 \\
\hline DL Metionina & 0.19 & 0.19 & 0.19 & 0.19 & 0.19 \\
\hline $\begin{array}{l}\text { Premezcla vitaminas }+ \\
\text { minerales }\end{array}$ & 0.10 & 0.10 & 0.10 & 0.10 & 0.10 \\
\hline Cloruro de colina 60 & 0.10 & 0.10 & 0.10 & 0.10 & 0.10 \\
\hline $\begin{array}{l}\text { Adsorvente de } \\
\text { micotoxinas }\end{array}$ & 0.10 & 0.10 & 0.10 & 0.10 & 0.10 \\
\hline Coccidiostato & 0.05 & 0.05 & 0.05 & 0.05 & 0.05 \\
\hline $\mathrm{HCl}$ Lisina & 0.08 & 0.08 & 0.08 & 0.08 & 0.08 \\
\hline Treonina & 0.01 & 0.01 & 0.01 & 0.01 & 0.01 \\
\hline Antioxidante & 0.02 & 0.02 & 0.02 & 0.02 & 0.02 \\
\hline Antibiótico & 0.05 & - & - & - & - \\
\hline Sulfato de cobre & - & - & 0.10 & - & - \\
\hline Oxicloruro de cobre & - & - & - & 0.05 & - \\
\hline ССТВ & - & - & - & - & 0.05 \\
\hline \multicolumn{6}{|c|}{ Valor Nutricional (Calculado) } \\
\hline $\begin{array}{l}\text { Energía metabolizable, } \\
\mathrm{kcal} \mathrm{kg}^{-1}\end{array}$ & 3,083 & 3,083 & 3,083 & 3,083 & 3,083 \\
\hline Proteín a bruta, \% & 19.00 & 19.00 & 19.00 & 19.00 & 19.00 \\
\hline Grasa total, $\%$ & 7.25 & 7.25 & 7.25 & 7.25 & 7.25 \\
\hline Fibra cruda, \% & 2.71 & 2.71 & 2.71 & 2.71 & 2.71 \\
\hline Calcio, \% & 0.96 & 0.96 & 0.96 & 0.96 & 0.96 \\
\hline Fósforo disponible, $\%$ & 0.48 & 0.48 & 0.48 & 0.48 & 0.48 \\
\hline Lisina total, \% & 1.10 & 1.10 & 1.10 & 1.10 & 1.10 \\
\hline Metionina total, $\%$ & 0.53 & 0.53 & 0.53 & 0.53 & 0.53 \\
\hline Met + Cis total, $\%$ & 0.84 & 0.84 & 0.84 & 0.84 & 0.84 \\
\hline Treonina total, $\%$ & 0.74 & 0.74 & 0.74 & 0.74 & 0.74 \\
\hline Triptófano, \% & 0.21 & 0.21 & 0.21 & 0.21 & 0.21 \\
\hline Sodio, \% & 0.17 & 0.17 & 0.17 & 0.17 & 0.17 \\
\hline
\end{tabular}


Cuadro 3. Composición porcentual y valor nutritivo de las dietas experimentales empleadas en la etapa de acabado (23 a 40 días)

\begin{tabular}{|c|c|c|c|c|c|}
\hline & \multicolumn{5}{|c|}{ Tratamientos ${ }^{1}$} \\
\hline & $\mathrm{T} 1$ & $\mathrm{~T} 2$ & $\mathrm{~T} 3$ & $\mathrm{~T} 4$ & T5 \\
\hline Maíz & 62.42 & 62.42 & 62.42 & 62.42 & 62.42 \\
\hline Torta de soya & 27.41 & 27.41 & 27.41 & 27.41 & 27.41 \\
\hline Aceite de soya & 6.00 & 6.00 & 6.00 & 6.00 & 6.00 \\
\hline Fosfato dicálcico & 1.91 & 1.91 & 1.91 & 1.91 & 1.91 \\
\hline Subproduc to de trigo & 0.28 & 0.28 & 0.28 & 0.28 & 0.28 \\
\hline Carbonato de calcio & 0.80 & 0.80 & 0.80 & 0.80 & 0.80 \\
\hline Sal común & 0.36 & 0.36 & 0.36 & 0.36 & 0.36 \\
\hline DL Metionina & 0.23 & 0.23 & 0.23 & 0.23 & 0.23 \\
\hline $\begin{array}{l}\text { Premezcla vitaminas }+ \\
\text { minerales }\end{array}$ & 0.10 & 0.10 & 0.10 & 0.10 & 0.10 \\
\hline Cloruro de colina 60 & 0.10 & 0.10 & 0.10 & 0.10 & 0.10 \\
\hline $\begin{array}{l}\text { Adsorvente de } \\
\text { micotoxinas }\end{array}$ & 0.10 & 0.10 & 0.10 & 0.10 & 0.10 \\
\hline Coccidiostato & 0.05 & 0.05 & 0.05 & 0.05 & 0.05 \\
\hline $\mathrm{HCl}$ Lisina & 0.14 & 0.14 & 0.14 & 0.14 & 0.14 \\
\hline Treonina & 0.05 & 0.05 & 0.05 & 0.05 & 0.05 \\
\hline Antioxidante & 0.02 & 0.02 & 0.02 & 0.02 & 0.02 \\
\hline Antibiótico & 0.05 & - & - & - & - \\
\hline Sulfato de cobre & - & - & 0.10 & - & - \\
\hline Oxicloruro de cobre & - & - & - & 0.05 & - \\
\hline ССТВ & - & - & - & - & 0.05 \\
\hline \multicolumn{6}{|c|}{ Valor Nutricional (Calculado) } \\
\hline $\begin{array}{l}\text { Energía metabolizable, } \\
\mathrm{kcal} \mathrm{kg}^{-1}\end{array}$ & 3,176 & 3,176 & 3,176 & 3,176 & 3,176 \\
\hline Proteín a bruta, \% & 18.00 & 18.00 & 18.00 & 18.00 & 18.00 \\
\hline Grasa total, \% & 8.83 & 8.83 & 8.83 & 8.83 & 8.83 \\
\hline Fibra cruda, \% & 2.72 & 2.72 & 2.72 & 2.72 & 2.72 \\
\hline Calcio, \% & 0.90 & 0.90 & 0.90 & 0.90 & 0.90 \\
\hline Fósforo disponible, \% & 0.45 & 0.45 & 0.45 & 0.45 & 0.45 \\
\hline Lisina total, \% & 1.05 & 1.05 & 1.05 & 1.05 & 1.05 \\
\hline Metionina total, \% & 0.52 & 0.52 & 0.52 & 0.52 & 0.52 \\
\hline Met + Cis total, \% & 0.82 & 0.82 & 0.82 & 0.82 & 0.82 \\
\hline Treonina total, \% & 0.72 & 0.72 & 0.72 & 0.72 & 0.72 \\
\hline Triptófano, \% & 0.21 & 0.21 & 0.21 & 0.21 & 0.21 \\
\hline Sodio, \% & 0.16 & 0.16 & 0.16 & 0.16 & 0.16 \\
\hline
\end{tabular}

${ }^{1}$ T1: dieta basal con antibiótico (control positivo), T2: dieta basal sin antibiótico (control negativo), T3: T2 + 250 ppm Cu como sulfato de cobre, T4: T2 + 250 ppm Cu como oxicloruro de cobre, T5: $\mathrm{T} 2+250$ ppm Cu como cloruro de cobre tribásico (CCTB)

to medio de cada vellosidad. La medida profundidad de cripta es la distancia tomada desde la región basal de cada vellosidad hasta la parte basal superior de la musculatura lisa del intestino. La relación altura de vellosidad y profundidad de cripta resultó de la división de ambas magnitudes. El área de la vellosidad fue calculada asumiendo una forma rectan- 
Cuadro 4. Efecto de la fuente de cobre sobre el comportamiento productivo de pollos de carne $^{2}$ (1 a 40 días de edad)

\begin{tabular}{|c|c|c|c|c|c|}
\hline \multirow{2}{*}{ Medición } & \multicolumn{5}{|c|}{ Tratamientos $^{1}$} \\
\hline & $\mathrm{T} 1$ & $\mathrm{~T} 2$ & $\mathrm{~T} 3$ & $\mathrm{~T} 4$ & $\mathrm{~T} 5$ \\
\hline Peso inicial, kg & $51.4 \pm 1.7^{2 \mathrm{a}}$ & $50.0 \pm 1.1^{\mathrm{a}}$ & $50.1 \pm 0.9^{\mathrm{a}}$ & $51.2 \pm 0.3^{\mathrm{a}}$ & $50.4 \pm 1.0^{\mathrm{a}}$ \\
\hline Peso final, $\mathrm{kg}$ & $2783 \pm 123^{\mathrm{a}}$ & $2770 \pm 158^{\mathrm{a}}$ & $2790 \pm 33^{\mathrm{a}}$ & $2887 \pm 86^{\mathrm{a}}$ & $2799 \pm 123^{\mathrm{a}}$ \\
\hline $\begin{array}{l}\text { Ganan cia de } \\
\text { peso g/día }\end{array}$ & $68.29 \pm 3.0^{\mathrm{a}}$ & $68.0 \pm 4.0^{\mathrm{a}}$ & $68.5 \pm 0.8^{\mathrm{a}}$ & $70.9 \pm 2.2^{\mathrm{a}}$ & $68.7 \pm 3.1^{\mathrm{a}}$ \\
\hline $\begin{array}{l}\text { Consumo de } \\
\text { alimento, g/día }\end{array}$ & $116.2 \pm 4.9^{\mathrm{a}}$ & $113.4 \pm 8.6^{\mathrm{a}}$ & $103.9 \pm 2.4^{\mathrm{b}}$ & $112.7 \pm 0.5^{\mathrm{a}}$ & $110.8 \pm 3.9^{\mathrm{ab}}$ \\
\hline $\begin{array}{l}\text { Conversión } \\
\text { alimenticia }\end{array}$ & $1.70 \pm 0.03^{\mathrm{a}}$ & $1.67 \pm 0.05^{\mathrm{ab}}$ & $1.52 \pm 0.04^{\mathrm{c}}$ & $1.57 \pm 0.04^{b c}$ & $1.62 \pm 0.12^{\mathrm{abc}}$ \\
\hline $\begin{array}{l}\text { Rendimiento } \\
\text { de carcasa, \% }\end{array}$ & $72.4 \pm 1.9^{\mathrm{a}}$ & $72.6 \pm 2.7^{\mathrm{a}}$ & $74.9 \pm 4.2^{\mathrm{a}}$ & $73.7 \pm 3.9^{\mathrm{a}}$ & $72.3 \pm 2.4^{\mathrm{a}}$ \\
\hline Mortalidad, \% & $20.0^{b}$ & $12.5^{\mathrm{ab}}$ & $5.0^{\mathrm{a}}$ & $17.5^{\mathrm{b}}$ & $5.0^{\mathrm{a}}$ \\
\hline
\end{tabular}

gular en donde la altura de la vellosidad multiplicada por el grosor de la vellosidad es igual al área. El número de células caliciformes fue la cuantificación total de estas células en cada una de las vellosidades seleccionadas. Por último, el promedio de las mediciones citomorfométricas de ocho láminas histológicas por segmento fue el promedio de estas magnitudes para cada tratamiento.

El microscopio óptico contaba con un ocular micrométrico. Las mediciones de altura y ancho de las vellosidades intestinales y profundidad de cripta se hicieron a 100x y el contaje de células caliciformes y de lámina propia a 400x. El cálculo de las mediciones se realizó utilizando un factor de corrección, multiplicando el factor del objetivo (0.010) por el número de líneas que abarca el tamaño del ocular micrométrico por 1000, dando el valor en micras $(\mu \mathrm{m})$. Con los resultados se calculó la relación altura de vellosidad y profundidad de cripta y el área de vellosidad.
Se empleó un diseño completamente al azar (Calzada, 1982) con cinco tratamientos y cuatro repeticiones por tratamiento. El análisis de varianza se llevó a cabo usando el programa Statistical Analysis System (SAS, 1999) y la comparación de medias se realizó utilizando la Prueba de Duncan (Calzada, 1982).

\section{Resultados y Discusión}

El Cuadro 4 muestra el efecto de la fuente de cobre sobre el comportamiento productivo de los pollos de 0 a 40 días de edad. Se observó una diferencia no significativa de $4 \%$ en el peso vivo en el día 40 a favor de $\mathrm{T} 4$ en relación a T1. Estos resultados guardan relación con otros estudios (Guo et al., 2001; Skrivan et al., 2002; Brainer et al., 2003), quienes tampoco encontraron diferencias estadísticas en el peso vivo de los pollos en el día 40 al suplementar con fuentes de cobre. 
Cuadro 5. Retribución económica del alimento por pollo de carne alimentado con dietas conteniendo diferentes fuentes de cobre

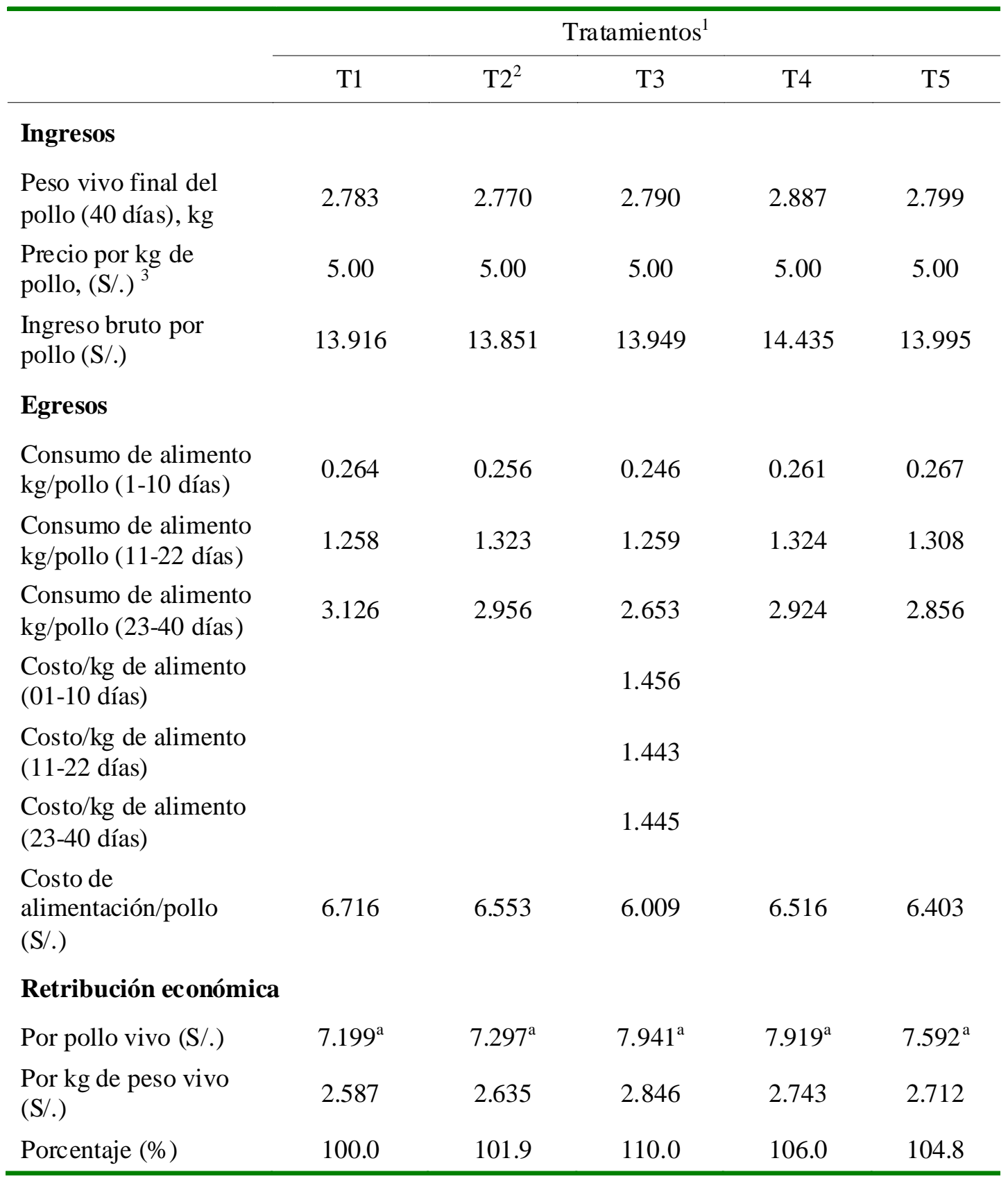

1 T1: dieta basal con antibiótico (control positivo), T2: dieta basal sin antibiótico (control negativo), T3: T2 + 250 ppm Cu como sulfato de cobre, T4: T2 + 250 ppm Cu como oxicloruro de cobre, T5: T2 + 250 ppm Cu como cloruro de cobre tribásico (CCTB)

2 Para efecto de la comparación relativa se considera como referencia a las aves alimentadas con la dieta basal sin antibiótico (T2)

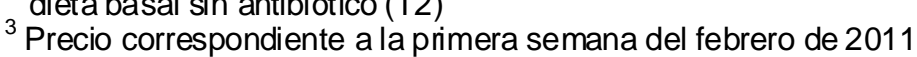

${ }^{a}$ Sin diferencia estadística $(p>0.05)$ 
Cuadro 6. Efecto de la fuente de cobre sobre la concentración de cobre en el hígado en base húmeda

\begin{tabular}{lc}
\hline Tratamientos & $\begin{array}{c}\text { Concentración de cobre } \\
(\mathrm{mg} / \mathrm{kg})\end{array}$ \\
\hline T1 - Dieta basal con antibiótico & $3.75 \pm 1.02^{\mathrm{b}}$ \\
T2 - Dieta basal sin antibiótico & $3.34 \pm 0.42^{\mathrm{b}}$ \\
T3 - Sulfato de cobre & $3.76 \pm 0.55^{\mathrm{b}}$ \\
T4 - Oxicloruro de cobre & $3.74 \pm 0.30^{\mathrm{b}}$ \\
T5 - Cloruro de cobre tribásico (CCTB) & $4.80 \pm 0.66^{\mathrm{a}}$ \\
\hline
\end{tabular}

${ }^{a, b}$ Superíndices diferentes indican diferencia estadística $(p<0.05)$

No se encontró diferencia estadística entre los dos grupos control (con y sin antibiótico) en ninguno de los parámetros de producción bajo evaluación. La literatura muestra un comportamiento productivo similar en algunas ocasiones entre animales con dietas con y sin antibióticos (Romero, 1998; Engberg et al., 2000; Gunal et al., 2006). Al respecto, Arias y Koutsos (2006) señalan que los antibióticos a nivel subterapéutico, en un medio ambiente con baja carga bacteriana, pueden afectar negativamente a las bacterias comensales necesarias para la apropiada digestión, resultando en una disminución del crecimiento. Por otro lado, hay reportes que demuestran los efectos benéficos del tratamiento con zinc bacitracina (Colin et al., 1994; Martínez, 1996).

La fuente de cobre tuvo un efecto significativo sobre el consumo de alimento, encontrándose un mayor consumo durante los 40 días del estudio en T4 sobre T3 ( $\mathrm{p}<0.05)$; no obstante, estas diferencias no afectaron las ganancias de peso totales entre tratamientos (Cuadro 4), aunque se llegó a encontrar una mayor ganancia de peso en el periodo de 0-21 días en T4 y T5 con el resto de grupos experimentales $(\mathrm{p}<0.05)$.

La fuente de cobre afectó la conversión alimenticia. Las aves de T3 tuvieron una mejor conversión alimenticia que los grupos control T1 y T2 (p<0.05), sin llegar a ser diferente a T4 y T5 para el periodo de 0-40 días; asimismo, T1 fue diferente a T4 $(p<0.05)$ (Cuadro 4). No se encontró diferencia estadística entre tratamientos en los periodos de 0 a 21,0 a 28 y 0 a 35 días.

Los grupos de aves en T3 y T5 presentaron la menor mortalidad $(\mathrm{p}<0.05)$. Cabe destacar que ocurrió un brote de infección gastrointestinal a partir del día 21 del estudio, afectando a todos los grupos experimentales; pese a ello se observó una mayor tendencia a resistir la infección en los animales de los tratamientos T3 y T5.

No se encontró diferencias estadísticas por efecto de los tratamientos en el rendimiento de carcasa. No obstante, Arias y Koutsos (2006) alimentando pollos con CCTB o sulfato de cobre obtuvieron mayores pesos de carcasa que los grupos control, con la diferencia que en ese trabajo las aves estuvieron alojadas en corrales y en el presente trabajo fue en baterías.

No se encontraron diferencias estadísticas entre tratamientos con respecto a la retribución económica (Cuadro 5), aunque se observó una tendencia favorable a las fuentes de cobre, especialmente en T3 (sulfato de cobre) sobre T2 en $8.1 \%$. 
Cuadro 7. Efecto de las fuentes de cobre sobre la morfometría intestinal

\begin{tabular}{|c|c|c|c|c|c|c|}
\hline Medidas & $\begin{array}{l}\text { Segmento del } \\
\text { intestino }\end{array}$ & $\mathrm{T} 1^{1}$ & $\mathrm{~T} 2$ & $\mathrm{~T} 3$ & $\mathrm{~T} 4$ & T5 \\
\hline $\begin{array}{l}\text { Altura de } \\
\text { vellosidad, } \\
\mu \mathrm{m}\end{array}$ & $\begin{array}{l}\mathrm{D}^{2} . \text { craneal } \\
\text { D. caudal } \\
\text { Yeyuno }\end{array}$ & $\begin{array}{c}1274 \pm 131^{\mathrm{a}} \\
1263 \pm 159^{\mathrm{a}} \\
985 \pm 201^{\mathrm{b}}\end{array}$ & $\begin{array}{l}1424 \pm 148^{\mathrm{a}} \\
1382 \pm 138^{\mathrm{a}} \\
1064 \pm 74^{\mathrm{ab}}\end{array}$ & $\begin{array}{c}1412 \pm 168^{\mathrm{a}} \\
1249 \pm 55^{\mathrm{a}} \\
1174 \pm 103^{\mathrm{a}}\end{array}$ & $\begin{array}{l}1326 \pm 184^{\mathrm{a}} \\
1286 \pm 124^{\mathrm{a}} \\
1175 \pm 131^{\mathrm{a}}\end{array}$ & $\begin{array}{l}1271 \pm 170^{\mathrm{a}} \\
1381 \pm 237^{\mathrm{a}} \\
1173 \pm 131^{\mathrm{a}}\end{array}$ \\
\hline $\begin{array}{l}\text { Ancho de } \\
\text { vellosidad, } \\
\mu \mathrm{m}\end{array}$ & $\begin{array}{l}\text { D. craneal } \\
\text { D. caudal } \\
\text { Yeyuno }\end{array}$ & $\begin{array}{c}195 \pm 27^{\mathrm{a}} \\
169 \pm 22^{\mathrm{ab}} \\
122 \pm 20^{\mathrm{a}}\end{array}$ & $\begin{array}{l}181 \pm 41^{\mathrm{a}} \\
185 \pm 25^{\mathrm{a}} \\
124 \pm 13^{\mathrm{a}}\end{array}$ & $\begin{array}{l}192 \pm 27 \mathrm{a} \\
178 \pm 27^{\mathrm{a}} \\
148 \pm 28^{\mathrm{a}}\end{array}$ & $\begin{array}{l}183 \pm 14^{\mathrm{a}} \\
148 \pm 12^{\mathrm{b}} \\
143 \pm 24^{\mathrm{a}}\end{array}$ & $\begin{array}{c}208 \pm 43^{\mathrm{a}} \\
169 \pm 36^{\mathrm{ab}} \\
149 \pm 28^{\mathrm{a}}\end{array}$ \\
\hline $\begin{array}{l}\text { Área de } \\
\text { vellosidad, } \\
\mu \mathrm{m}^{2}\end{array}$ & $\begin{array}{l}\text { D. craneal } \\
\text { D. caudal } \\
\text { Yeyuno }\end{array}$ & $\begin{array}{c}26476^{\mathrm{a}} \\
21236^{\mathrm{ab}} \\
12162^{\mathrm{b}}\end{array}$ & $\begin{array}{c}23836^{\mathrm{a}} \\
25075^{\mathrm{a}} \\
13446^{\mathrm{ab}}\end{array}$ & $\begin{array}{l}26246^{\mathrm{a}} \\
21448^{\mathrm{ab}} \\
17453^{\mathrm{a}}\end{array}$ & $\begin{array}{l}24789^{\mathrm{a}} \\
18476^{\mathrm{b}} \\
16957^{\mathrm{a}}\end{array}$ & $\begin{array}{c}23353^{\mathrm{a}} \\
23943^{\mathrm{ab}} \\
17886^{\mathrm{a}}\end{array}$ \\
\hline $\begin{array}{l}\text { Profundidad } \\
\text { de cripta, } \mu \mathrm{m}\end{array}$ & $\begin{array}{l}\text { D. craneal } \\
\text { D. caudal } \\
\text { Yeyuno }\end{array}$ & $\begin{array}{l}179 \pm 27^{\mathrm{a}} \\
182 \pm 19^{\mathrm{a}} \\
140 \pm 19^{\mathrm{a}}\end{array}$ & $\begin{array}{c}186 \pm 39.0^{\mathrm{a}} \\
179 \pm 27^{\mathrm{a}} \\
125 \pm 17^{\mathrm{a}}\end{array}$ & $\begin{array}{l}159 \pm 20^{\mathrm{a}} \\
175 \pm 30^{\mathrm{a}} \\
132 \pm 26^{\mathrm{a}}\end{array}$ & $\begin{array}{l}183 \pm 17^{\mathrm{a}} \\
185 \pm 30^{\mathrm{a}} \\
146 \pm 35^{\mathrm{a}}\end{array}$ & $\begin{array}{c}1780 \pm 13^{\mathrm{a}} \\
179 \pm 24^{\mathrm{a}} \\
139 \pm 37^{\mathrm{a}}\end{array}$ \\
\hline $\begin{array}{l}\text { Células } \\
\text { caliciformes, } \\
\text { N. }{ }^{\circ}\end{array}$ & $\begin{array}{l}\text { D. craneal } \\
\text { D. caudal } \\
\text { Yeyuno }\end{array}$ & $\begin{array}{c}83 \pm 21^{\mathrm{a}} \\
82 \pm 20^{\mathrm{a}} \\
144 \pm 74^{\mathrm{a}}\end{array}$ & $\begin{array}{c}90 \pm 22^{\mathrm{a}} \\
82 \pm 11.3^{\mathrm{a}} \\
116 \pm 37^{\mathrm{a}}\end{array}$ & $\begin{array}{c}77 \pm 13^{\mathrm{a}} \\
79 \pm 10^{\mathrm{a}} \\
172 \pm 62^{\mathrm{a}}\end{array}$ & $\begin{array}{c}76 \pm 5^{\mathrm{a}} \\
81 \pm 23^{\mathrm{a}} \\
147 \pm 27^{\mathrm{a}}\end{array}$ & $\begin{array}{c}85 \pm 47^{\mathrm{a}} \\
88 \pm 43^{\mathrm{a}} \\
153 \pm 102^{\mathrm{a}}\end{array}$ \\
\hline $\begin{array}{l}\text { Ancho de } \\
\text { lámina } \\
\text { propia, } \mu \mathrm{m}\end{array}$ & $\begin{array}{l}\text { D. craneal } \\
\text { D. caudal } \\
\text { Yeyuno }\end{array}$ & $\begin{array}{l}5.6 \pm 0.4^{\mathrm{a}} \\
4.4 \pm 0.8^{\mathrm{a}} \\
4.7 \pm 0.8^{\mathrm{a}}\end{array}$ & $\begin{array}{l}5.3 \pm 1.4^{\mathrm{a}} \\
4.7 \pm 0.5^{\mathrm{a}} \\
4.4 \pm 0.6^{\mathrm{a}}\end{array}$ & $\begin{array}{l}5.3 \pm 1.1^{\mathrm{a}} \\
4.9 \pm 1.6^{\mathrm{a}} \\
5.3 \pm 1.0^{\mathrm{a}}\end{array}$ & $\begin{array}{l}5.3 \pm 0.8^{\mathrm{a}} \\
4.1 \pm 1.1^{\mathrm{a}} \\
4.4 \pm 0.5^{\mathrm{a}}\end{array}$ & $\begin{array}{l}5.2 \pm 1.0^{\mathrm{a}} \\
4.7 \pm 1.0^{\mathrm{a}} \\
4.9 \pm 0.9^{\mathrm{a}}\end{array}$ \\
\hline $\begin{array}{l}\text { Relación } \\
\text { altura/ } \\
\text { profundidad }\end{array}$ & $\begin{array}{l}\text { D. craneal } \\
\text { D. caudal } \\
\text { Yeyuno }\end{array}$ & $\begin{array}{l}7.3 \pm 1.4^{\mathrm{a}} \\
7.0 \pm 1.1^{\mathrm{a}} \\
7.2 \pm 1.8^{\mathrm{a}}\end{array}$ & $\begin{array}{l}7.8 \pm 1.1^{\mathrm{a}} \\
7.8 \pm 1.2^{\mathrm{a}} \\
8.7 \pm 1.4^{\mathrm{a}}\end{array}$ & $\begin{array}{l}9.0 \pm 1.7^{\mathrm{a}} \\
7.3 \pm 1.3^{\mathrm{a}} \\
9.1 \pm 1.2^{\mathrm{a}}\end{array}$ & $\begin{array}{l}7.3 \pm 0.8^{\mathrm{a}} \\
7.1 \pm 1.2^{\mathrm{a}} \\
8.3 \pm 1.5^{\mathrm{a}}\end{array}$ & $\begin{array}{l}7.3 \pm 1.6^{\mathrm{a}} \\
7.8 \pm 1.6^{\mathrm{a}} \\
9.0 \pm 2.7^{\mathrm{a}}\end{array}$ \\
\hline
\end{tabular}

La concentración de cobre en el hígado se presenta en el Cuadro 6. Solo se encontró diferencia significativa favorable a T5 $(\mathrm{p}<0.05)$, a diferencia del trabajo de Ewing et al. (1998) quienes encontraron hasta en un $26 \%$ de incremento de niveles de cobre en el hígado usando sulfato de cobre pentahidratado y oxicloruro de cobre. La mayor cantidad de cobre se debería a la mayor biodisponibilidad (Luo et al., 2005). Asimismo, la mayor concentración en T5 indica que esta fuente (cobre clorado tribásico) se almacena mejor en el hígado que otras fuentes de cobre.

La suplementación con cobre tuvo efecto favorable en la altura de vellosidad del yeyuno en los tratamientos T3, T4 y T5 sobre $\mathrm{T} 1 \mathrm{y}$ en el área de vellosidad del yeyuno en T3, T4 y T5 sobre T1 ( $\mathrm{p}<0.05)$ (Cuadro 7). Estos resultados coinciden con Xia et al. (2004) y Arias y Koutsos (2006), quienes 
señalan que fuentes de cobre, como sulfato de cobre, CCTB y Cu-MMT, afectan diversas áreas del intestino delgado, haciendo evidente una relación positiva entre las medidas morfométricas del intestino con la ganancia de peso y los índices de consumo y conversión alimenticia.

A diferencia del yeyuno, no se encontraron diferencias estadísticas para las medidas citomorfométricas entre los tratamientos para los otros segmentos del intestino, con excepción del ancho de vellosidad del duodeno caudal entre T4 con T2 y T3 (Cuadro 7). La mayor anchura de las vellosidades podría ser a consecuencia de una hiperplasia por la infección gastrointestinal que ocurrió alrededor del día 21 del experimento, de allí que los tratamientos con menor ancho de vellosidad como el T4 y T5 estarían denotando un efecto protector de las fuentes de cobre sobre el intestino. Al respecto, Arias y Koutsos (2006) encontraron una menor cantidad de linfocitos en el duodeno de animales suplementados con cobre, en relación a un control negativo sin suplementación.

Por último, no hubo diferencia estadística entre tratamientos con relación al número de células caliciformes, ancho de la lámina propia y relación altura/profundidad de cripta.

\section{Conclusiones}

- Las fuentes de cobre no tuvieron influencia significativa sobre la ganancia de peso de los pollos de carne; sin embargo, la adición de cobre en forma de sulfato de cobre mejoró la conversión y el consumo.

- La adición de cobre en forma de cobre clorado tribásico resultó en un mayor depósito de cobre en el hígado.
- La altura y el área de las vellosidades del yeyuno fueron influenciadas por la fuente de cobre en la dieta.

\section{Agradecimilento}

Los autores agradecen el apoyo técnico del Dr. Alfonso Chavera Castillo y de la Dra. Rosa Perales Camacho, del Laboratorio de Histopatología, Facultad de Medicina Veterinaria, Universidad Nacional Mayor de San Marcos.

\section{Literatura Citada}

1. Abaza IM, Ezzat W, Shoeib MS, Eizaiat AA, Hassan II. 2009. Effects of copper sulfate on productive, reproductive performance and blood constituents of laying Japanese quail fed optimal and sub-optimal protein. Int $\mathbf{J}$ Poultry Sci 1: 80-89.

2. Arias VJ, Koutsos EA. 2006. Effects of copper source and level on intestinal physiology and growth of broiler chickens. Poultry Sci 85: 999-1007.

3. Batista de Oliveira P, Murakami AE, De Moraes García ER, Macari M, Scapinello C. 2000. Influence of antinutritional factors of leucaena (Leucaena leucocephala and Leucaena cunningan) and pigeous bean (Cajanus cajan) on the intestinal epithelium and performance of broiler chickens. Rev Bras Zootec 29: 17591769.

4. Brainer MM, Machado JF, Martínez M, Daroz SC. 2003. Cupric citrate as growth promoter for broiler chickens in different rearing stages. Scientiae Agricola 3: 441-445.

5. Calzada J. 1982. Métodos estadísticos para la investigación. Lima: Ed Jurídica. $644 \mathrm{pp}$. 
6. Cobb-Vantress. 2002. Cobb 500 Breeder management guide. CobbVantress Inc., Siloam Springs, AR. Available in: http://www.cobb-vantress.com/docs/default-source/cobb500-guides/cobb500_bpn_supp_spanish.pdf?sfvrsn=2

7. Colin L, Morales E, Ávila E. 1994. Evaluación de promotores de crecimiento para pollos de engorda. Vet Méx 25: 141144.

8. Desmonts $\mathrm{MH}$, Dufour-Gesbert $\mathrm{F}$, Avrain L, Kempf I. 2004. Antimicrobial resistance in Campylobacter strains isolated from French broilers before and after antimicrobial growth promoters bans. J Antimicrob Chemoth 54: 10251030.

9. Enberg RM, Hederann MS, Leser TD, Jensen BB. 2000. Effect of zinc bacitracin and salinomycin on intestinal microflora and performance of broiler. Poultry Sci 79: 1311-1319.

10. Ewing HP, Pesti GM, Bakall RI, Menten JF. 1998. Studies on the feeding of cupric sulfate pentahydrate, cupric citrate, and copper oxychloride to broiler chickens. Poultry Sci 77: 445-448.

11. Gunal M, Yayli G Kaya O, Karahan $N$, Sulak O. 2006. The effects of antibiotic growth promoter, probiotic or organic acid supplementation on performance, intestinal microflora and tissue of broilers. Int J Poultry Sci 2: 149-155.

12. Guo R, Henry PR, Holwenda RA, Cao $J$, Little $R C$, Miles RD, Ammerman CB. 2001. Chemical characteristics and relative bioavailability of supplemental organic copper sources for poultry. J Anim Sci 79: 1132-1141.

13. Ledoux DR, Henry PR, Ammerman CB, Rao PV, Miles R.D. 1991. Estimation of the relative bioavailability of inorganic copper sources for chicks using tissue uptake of copper. J Anim Sci 69: 215-222.

14. Luo XG, Ji F, Lin YX, Steward FA, Lu L, Liu B, Yu SX. 2005. Effects of dietary supplementation with copper sulfate or tribesic copper chloride on broiler performance, relative copper bioavailability, and oxidation stability of vitamin $E$ in feed. Poultry Sci 84: 888-893.

15. Martínez M. 1996. Efecto de la avilamicina, avoparcina, y zinc cacitracina en el rendimiento productivo de pollos de carne. Lima: UNALM. 55 p.

16. Mejía OR, Ruiz M, Grimaldi DC, García GA, Ruiz AL, García A, Casadiego CA. 2006. Bases biológicas y patobiológicas humanas del metabolismo del cobre. Universitas Médica 1: 5572.

17. Miles RD, O'Keefe SF, Henry PR, Ammerman CB, Luo XG. 1998. The effect of dietary supplementation with copper sulfate or tribasic copper chloride on broiler performance, relative copper bioavailability, and dietary prooxidant activity. Poultry Sci 77: 416-425.

18. Miñón E, Gonzales MJ, Huerta M, Crespo G, Carrillo S, Castillo RM, Cuca JM, Morales E. 2006. Niveles óptimos biológico y económico de cobre dietético en pollos de engorda. Agrociencia 40: 163-170.

19. [NRC] National Research Council. 1994. Nutrient requirements of poultry. $9^{\text {th }}$ ed. Washington DC: National Academy Press.

20. Norma Oficial Mexicana NOM-117SSA1-1994. Bienes y servicios. 1994. Método de prueba para la determinación de cadmio, arsénico, plomo, estaño, cobre, fierro, zinc y mercurio en alimentos, agua potable y agua purificada por espectrometría de absorción atómica. [Internet]. Disponible en: http:// www.salud.gob.mx/unidades/cdi/nom/ 117ssa14.html

21. Pesti G, Bakalli R. 1998. Studies on the effect of feeding cupric sulfate pentahidrate to laying hens on egg cholesterol content. Poultry Sci 77: 15401545 .

22. Romero CA. 1998. Evaluación comparativa de la suplementación de flavosfolipol, olaquindox y zinc bacitracina en la alimentación de pollos 
de carne. Lima: Univ Nacional Agraria La Molina. $74 \mathrm{p}$.

23. [SAS] Statistical Analysis System. 1999. SAS user's guide: statistics. Cary: SAS institute. $965 \mathrm{p}$.

24. Skrivan M, Sevcikova E, Tumova E, Skrivanova V, Marounek M. 2002. Effect of copper sulphate supplementation on performance of broiler chickens, cholesterol content and fatty acid profile of meat. Czech J Anim Sci 47:275-280.
25. van den Bogaard A, Stobberingh E. 2000. Epidemiology of resistance toantibiotics. Links between animals and humans. Int J Antimicrob Agents 14: 327335.

26. Xia MS, Hu CH, Xu ZR. 2004. Effects of copper-bearing montmorillonite on growth performance, digestive enzyme activities, and intestinal microflora and morphology of male broilers. Poultry Sci 83: 1868-1875. 